\title{
Theoretical analysis of the delay on the p53 micronetwork
}

\author{
Danni Wang ${ }^{1}$, Nan Liu', Hongli Yang ${ }^{1 *}$ (I) and Liangui Yang
}

"Correspondence:

hongliyang3@sohu.com

${ }^{1}$ School of Mathematical Sciences, Inner Mongolia University, Hohhot, 010021, China

\section{Springer}

\begin{abstract}
Time delay plays a crucial role in p53 dynamic. However, the theoretical understanding is still lower. Thus we construct a micro-differential equation model and introduce the time delay $\tau$ based on the regulation process. Firstly, we linearize the system and analyze the associated characteristic equation. We can conclude that there exists the delay threshold $\tau_{0}$ such that when the delay $\tau$ is less than $\tau_{0}$, the system is asymptotically stable and otherwise stable oscillations occur. Secondly, we use the normal form method and the center manifold theorem to derive the direction and stability of the Hopf bifurcation. Finally, by numerical simulations we verify our theoretical results. We also find that the effect of noise on the amplitude is more severe than that of the period, which well agrees with the experimental results.
\end{abstract}

Keywords: Delays; Oscillations; Hopf bifurcation

\section{Introduction}

The tumor suppressor p53 is located in the center of the cellular signaling networks and stress-activated signals networks [1]. In unstressed cells, wild-type p53 remains at low levels [2]. Upon DNA damage, p53 is stabilized and activated [3, 4]. p53 can induce different cellular outcomes such as cell cycle arrest and apoptosis [5-12]. It is very crucial to study the mechanism of p53 network dynamics [13].

Recently, it was experimentally shown that the concentration of p53 undergoes pulses upon ionization radiation in MCF7 cells [14]. Much work has explored the feedback mechanism of p53 oscillations $[6,7,15,16]$. It has been suggested that the fate of cells between survival and death can be determined by counting the number of p53 pulses: cells can survive on the transient p53 pulses, but apoptosis under the action of continuous p53 pulse [7-10]. This may represent a reliable and flexible mechanism. For example, it can prevent premature apoptosis caused by drastic unexpected fluctuations in p53 levels [17].

The p53-Mdm2 negative feedback loop is recognized as the basis of p53 oscillation $[6,18]$, and the research has shown that the ATM (ataxia telangiectasia mutated)-p53WIP1(wild-type p53-induced phosphatase1) negative feedback loop (NFL) is required for the generation of uniform p53 pulses [16]. Therefore it is important to further clarify how p53 pulses are regulated.

(c) The Author(s) 2020. This article is licensed under a Creative Commons Attribution 4.0 International License, which permits use sharing, adaptation, distribution and reproduction in any medium or format, as long as you give appropriate credit to the original author(s) and the source, provide a link to the Creative Commons licence, and indicate if changes were made. The images or other third party material in this article are included in the article's Creative Commons licence, unless indicated otherwise in a credit line to the material. If material is not included in the article's Creative Commons licence and your intended use is not permitted by statutory regulation or exceeds the permitted use, you will need to obtain permission directly from the copyright holder. To view a copy of this licence, visit http://creativecommons.org/licenses/by/4.0/. 
Figure 1 Schematic depiction of the model. p53 promotes the transcription of MDM2, which in turn inhibits p53 through ubiquitination, forming a negative feedback loop of p53-MDM2; Activated ATM promotes the stability and activation of $\mathrm{p} 53$, which in turn inactivates ATM, forming a negative feedback loop of p53-ATM. The promotion and inhibition of state transition are separately denoted by arrow-headed solid lines and bar-headed lines, and $\tau$ indicates the delay of p53 on promoting Mdm2 or inhibiting ATM

Mathematical models are generally used to study dynamic processes [19], including, from the cell process point of view, a series of regulatory behaviors in biological cells, such as transcriptional translation, transmembrane transport, and so on [20-23]. All they possess a certain time delay $[6,10,24,25]$. We construct p53-MDM2-ATM micro network with time delays to fit the biological facts. Based on the real networks modeling and theoretical research, we analyze the influence of time delay on the oscillation behavior of the simplified model and verify the correctness of the theoretical analysis by numerical simulations.

\section{Models and assumptions}

In resting cells, p53 is suppressed by MDM2 at low level [26]. When DNA is damaged by external stimulation, DNA double-strand breaks (DSBs) promote the activation of ATM, which make p53 transform to active state [6,27]. Activated p53 is beneficial to the transcription of p53 target protein. WIP1 is one of the proteins that promoted by $\mathrm{p} 53[5,9]$ can catalyze the dephosphorylation of ATM [28]. WIP1-ATM-p53 forms an NFL. To avoid the complexity of the model, we hide the WIP1 protein, that is, p53 can directly inhibit ATM with time delay $\tau$. At the same time, p53 promotes the production of MDM2 in cytoplasm (MDM2c) [29]. When MDM2c is phosphorylated, it moves to the nucleus [29]. MDM2n (MDM2 in nucleus) in turn promotes the ubiquitin of p53, accelerates the degradation of p53 [9], and encloses the p53-MDM2 NFL. Due to the existence of transmembrane transport, here we add a time delay to the effect of p53 on MDM2. To simplify the numerical calculation and theoretical analysis, we choose the same time delay as shown in Fig. 1.

Through p53 mininetwork, we give system (1), where $\beta$ is the phosphorylation rate constant of ATM under DSBS, $\gamma$ and $\alpha$ are the maximum production rate constants of $\mathrm{p} 53$ and MDM2, respectively, $\zeta$ is the production rate of noise in protein. For simplicity, we take $\zeta=1$ in the next theory study, that is, the ideal cell environment. $\eta$ is the dephosphorylation rate constant of ATM dependent on p53, and $k \eta$ is the basal ATM dephosphorylation rate. We use $A(t), P(t), M(t)$ to denote the concentrations of ATM, p53, and Mdm2, respectively. All they are dimensionless concentrations. $k_{i}(i=1,2,3,4)$ are Michaelis constants of the Michaelis-Menten function or Hill function.

$$
\left\{\begin{array}{l}
\dot{A}(t)=\beta-\eta(P(t-\tau)+k) \frac{A(t)}{k_{1}+A(t)}, \\
\dot{P}(t)=\zeta \gamma \frac{A(t)}{k_{2}+A(t)}-u M(t) \frac{P(t)}{k_{3}+P(t)}, \\
\dot{M}(t)=\zeta \alpha \frac{P^{4}(t-\tau)}{k_{4}^{4}+P^{4}(t-\tau)}-\mu \frac{A(t)}{k_{2}+A(t)} M(t) .
\end{array}\right.
$$

\section{Local stability and Hopf bifurcation}

In this part, we theoretically analyze the bifurcation and oscillation mechanism of system (1) under time delay. The equilibrium point of the system is $\hat{E}=\left(A^{*}, P^{*}, M^{*}\right)$. Let 
$\bar{A}(t)=A(t)-A^{*}, \bar{P}(t)=P(t)-P^{*}, \bar{M}(t)=M(t)-M^{*}$. For simplicity, we still denote $\bar{A}, \bar{P}, \bar{M}$ by $A, P, M$. System (1) is deployed using Taylor's formula. We obtain

$$
\left\{\begin{aligned}
\dot{A}(t)= & C_{1} A(t)+C_{2} P(t-\tau)-\eta P(t-\tau) G^{\prime}\left(A^{*}\right) A(t) \\
& -\eta\left(P(t-\tau)+P^{*}+k\right) \sum_{i=2}^{\infty} \frac{1}{i !} G^{i}\left(A^{*}\right) A^{i}(t), \\
\dot{P}(t)= & C_{3} A(t)+C_{4} P(t)+C_{5} M(t)-u P(t) M(t) f^{\prime}\left(P^{*}\right) \\
& +\gamma \sum_{i=2}^{\infty} \frac{1}{i !} G_{1}{ }^{i}(A *) A^{i}(t)-u\left(M(t)+M^{*}\right) \sum_{i=2}^{\infty} \frac{1}{i !} G^{i}(A *) A^{i}(t), \\
\dot{M}(t)= & C_{6} A(t)+C_{7} P(t-\tau)+C_{8} M(t)-\mu A(t) M(t) G_{1}{ }^{\prime}\left(A^{*}\right) \\
& +\alpha \sum_{i=2}^{\infty} \frac{1}{i} f_{2}{ }^{i}\left(P^{*}\right) P^{i}(t-\tau)+\left(M(t)+M^{*}\right) \sum_{i=2}^{\infty} \frac{1}{i !} G_{1}{ }^{i}\left(A^{*}\right) A^{i}(t),
\end{aligned}\right.
$$

where superscript $i$ represents the $i$ th-order derivative, and

$$
\begin{aligned}
& C_{1}=-\eta\left(P^{*}+k\right) G^{\prime}\left(A^{*}\right), \quad C_{2}=-\eta G\left(A^{*}\right), \quad C_{3}=\gamma G^{\prime}\left(A^{*}\right), \\
& C_{4}=-u M^{*} f^{\prime}\left(P^{*}\right), \quad C_{5}=-u f\left(P^{*}\right), \quad C_{6}=-\mu M^{*} G^{\prime}\left(A^{*}\right), \\
& C_{7}=\alpha f_{2}^{\prime}\left(P^{*}\right), \quad C_{8}=-\mu G_{1}\left(A^{*}\right), G^{\prime}(A)=\frac{k_{1}}{\left(k_{1}+A^{*}\right)^{2}}, \\
& f^{\prime}(P)=\frac{k_{3}}{\left(k_{3}+P^{*}\right)^{2}}, \quad G_{1}^{\prime}(A)=\frac{k_{2}}{\left(k_{2}+A^{*}\right)^{2}}, \\
& f_{2}^{\prime}(P)=\frac{4 k_{4}^{4} P^{3}(t-\tau)}{\left(k_{4}^{4}+P^{4}(t-\tau)\right)^{2}} .
\end{aligned}
$$

The stable point $\hat{E}$ of system (1) is transformed into zero $E^{0}(0,0,0)$ of system (2), and we can get the linearized system

$$
\left\{\begin{array}{l}
\dot{A}(t)=C_{1} A(t)+C_{2} P(t-\tau) \\
\dot{P}(t)=C_{3} A(t)+C_{4} P(t)+C_{5} M(t) \\
\dot{M}(t)=C_{6} A(t)+C_{7} P(t-\tau)+C_{8} M(t)
\end{array}\right.
$$

and then we get the characteristic equation

$$
\lambda^{3}+A_{1} \lambda^{2}+A_{2} \lambda+a_{0}+a_{1} e^{-\lambda \tau} \lambda+a_{2} e^{-\lambda \tau}=0
$$

where

$$
\begin{array}{ll}
A_{1}=-\left(C_{1}+C_{4}+C_{8}\right), & A_{2}=-\left(C_{1} C_{8}+C_{4} C_{8}-C_{1} C_{4}\right), \quad a_{0}=-C_{1} C_{4} C_{8}, \\
a_{1}=-\left(C_{2} C_{3}+C_{5} C_{7}\right), & a_{2}=C_{2} C_{3} C_{8}+C_{1} C_{5} C_{7}-C_{2} C_{5} C_{6} .
\end{array}
$$

To obtain all the cases from the theoretical point of view, we assume that $i \omega(\omega>0)$ is the root of Eq. (4) and $\omega$ satisfies the equation

$$
\begin{aligned}
& -i \omega^{3}-A_{1} \omega^{2}+A_{2} i \omega+a_{0}+a_{1} i \omega(\cos (\omega \tau)-i \sin (\omega \tau)) \\
& +a_{2}(\cos (\omega \tau)-i \sin (\omega \tau))=0 .
\end{aligned}
$$


The real and imaginary parts of Eq. (5) can be separated from each other, and we obtain

$$
\left\{\begin{array}{l}
-A_{1} \omega^{2}+a_{0}+a_{1} \omega \sin (\omega \tau)+a_{2} \omega \cos (\omega \tau)=0, \\
-i \omega^{3}+A_{2} i \omega+a_{1} i \omega \cos (\omega \tau)-a_{2} i \omega \sin (\omega \tau)=0
\end{array}\right.
$$

which leads to

$$
a_{1}^{2} \omega^{2}+a_{2}^{2} \omega^{2}=\left(A_{1} \omega^{2}-a_{0}\right)^{2}+\left(\omega^{3}-A_{2} \omega\right)^{2} .
$$

Then we get the equation

$$
b_{0}+b_{1} \omega^{2}+b_{2} \omega^{4}+\omega^{6}=0
$$

where

$$
b_{0}=a_{0}^{2}, \quad b_{1}=A_{2}^{2}-a_{1}^{2}-a_{2}^{2}-2 a_{0} A_{1}, \quad b_{2}=A_{1}^{2}-2 A_{2} .
$$

If there is at least one positive real root of Eq. (8), then Eq. (4) has a pair of purely imaginary roots at the critical value of $\tau$. We define that the positive root of Eq. (8) is $\omega_{l}, 1 \leq l \leq 6$. For every fixed $l$, the corresponding critical value of time delay $\tau$ is

$$
\begin{aligned}
\tau_{l}^{(j)} & =\frac{2 j \pi}{\omega_{l}}+\frac{1}{\omega_{l}} \times \arccos \left[\frac{\left.\left(A_{1} \omega^{2}-a_{0}\right) a_{2}+\omega^{3} a_{1}-A_{2} a_{1} \omega^{2}\right)}{a_{1}^{2} \omega^{2}+a_{2}^{2}}\right] \\
1 & \leq l \leq 6, j=0,1,2, \ldots .
\end{aligned}
$$

For simplicity, we record that the minimum value of $\tau_{l}^{(j)}$ is represented by $\tau_{0}$. When $\tau_{l}=$ $\tau_{0}$, the corresponding $\omega_{l}=\omega_{0}$. Let $\lambda(\tau)=\nu(\tau)+i \omega(\tau)$ be the root of Eq. (4) that satisfies $v\left(\tau_{0}\right)=0$ and $\omega\left(\tau_{0}\right)=0$. We can prove that $\left.[d(\operatorname{Re}(\lambda) / d \tau)]\right|_{\tau=\tau_{0}}>0$.. Substituting $\lambda(\tau)$ into the left-hand side of the Eq. (4), we obtain

$$
\begin{aligned}
\left(\frac{d(\operatorname{Re}(\lambda))}{d \tau}\right)^{-1} & =\operatorname{Re}\left[\frac{\left(3 \lambda^{2}+2 A_{1} \lambda+A_{2}\right) e^{\lambda \tau}+a_{1}}{-a_{1} \lambda^{2}-a_{2} \lambda}\right] \\
& =\frac{W_{0} R_{1}+W_{0}^{2} R_{2}+W_{0}^{3} R_{3}+W_{0}^{4} R_{4}}{B_{1}^{2}+B_{2}^{2}},
\end{aligned}
$$

where

$$
\begin{aligned}
& R_{1}=-A_{2} a_{2} \sin \left(\omega_{0} \tau_{0}\right), \quad R_{2}=A_{2} a_{1} \cos \left(\omega_{0} \tau_{0}\right)-2 A_{1} a_{2} \cos \left(\omega_{0} \tau_{0}\right)-2 A_{1} a_{2} \sin \left(\omega_{0} \tau_{0}\right) \\
& R_{3}=3 a_{2} \sin \left(\omega_{0} \tau_{0}\right), \quad R_{4}=-3 \cos \left(\omega_{0} \tau_{0}\right), \quad B_{1}=a_{1} \omega_{0}^{2}, \quad B_{2}=a_{2} \omega_{0} .
\end{aligned}
$$

Obviously, when $\omega_{0} R_{1}+\omega_{0}^{2} R_{2}+\omega_{0}^{3} R_{3}+\omega_{0}^{4} R_{4}>0$, we have

$$
\operatorname{sign}\left\{\left.\left[\frac{d(\operatorname{Re}(\lambda))}{d \tau}\right]\right|_{\tau=\tau_{0}}\right\}=\operatorname{sign}\left\{\left.\operatorname{Re}\left[\frac{d(\lambda)}{d \tau}\right]^{-1}\right|_{\tau=\tau_{0}}\right\}>0 .
$$


Therefore the conclusion is that when $0 \leq \tau<\tau_{0}$, the equilibrium point $\hat{E}$ of system (1) is asymptotically stable; when $\tau>\tau_{0}$, the system is unstable; obviously, when $\tau=\tau_{0}$, the system experiences Hopf bifurcation at the equilibrium point. From this conclusion we can clearly realize that $\tau_{0}$ is a very important value; when the system delay is greater than $\tau_{0}$, the system is oscillatory, and otherwise it remains stable. The influence mechanism of time delay on the oscillation of p53-MDM2-ATM system can be obtained by studying $\tau_{0}$.

\section{Direction and stability of the Hopf bifurcation}

In this section, we continue to study the Hopf bifurcation through the normal form theory and the center manifold reduction theory. Define $\tau=\gamma+\tau_{0}$. When $\gamma=0$, as in the third part of the study, we normalize the time scale by $t \rightarrow\left(\frac{t}{\tau}\right)$, rewriting the system as follows:

$$
\left\{\begin{aligned}
\dot{A}(t)= & \left(\tau_{0}+\gamma\right)\left[C_{1} A(t)+C_{2} P(t-\tau)-\eta P(t-\tau) G^{\prime}(A *) A(t)\right. \\
& \left.-\eta\left(P(t-\tau)+P^{*}+k\right) \sum_{i=2}^{\infty} \frac{1}{i !} G^{i}(A *) A^{i}(t)\right], \\
\dot{P}(t)= & \left(\tau_{0}+\gamma\right)\left[C_{3} A(t)+C_{4} P(t)+C_{5} M(t)-u P(t) M(t) f^{\prime}\left(P^{*}\right)\right. \\
& \left.+\gamma \sum_{i=2}^{\infty} \frac{1}{i !} G_{1}{ }^{i}(A *) A^{i}(t)-u\left(M(t)+M^{*}\right) \sum_{i=2}^{\infty} \frac{1}{i !} G^{i}(A *) A^{i}(t)\right], \\
\dot{M}(t)= & \left(\tau_{0}+\gamma\right)\left[C_{6} A(t)+C_{7} P(t-\tau)+C_{8} M(t)-\mu A(t) M(t) G_{1}{ }^{\prime}(A *)\right. \\
& \left.+\alpha \sum_{i=2}^{\infty} \frac{1}{i !} f_{2}{ }^{i}(P *) P^{i}(t-\tau)+\left(M(t)+M^{*}\right) \sum_{i=2}^{\infty} \frac{1}{i !} G_{1}{ }^{i}(A *) A^{i}(t)\right] .
\end{aligned}\right.
$$

Let $U=(A(t), P(t), M(t))^{T}$ and $U_{t}=U(t+\theta), \theta \in[-1,0]$. We denote $C=C\left([-1,0], R^{3}\right)$. Then system (10) can be transformed to

$$
\dot{U}=L_{\gamma}\left(U_{t}\right)+f\left(\gamma, U_{t}\right)
$$

Equation (12) can be obtained by defining the linear operator $L_{\gamma}: C \rightarrow R^{3}$ and the nonlinear operator $f: R \times C \rightarrow R^{3}$ as follows:

$$
L_{\gamma}(\phi)=\left(\tau_{0}+\gamma\right)\left(\begin{array}{ccc}
C_{1} & 0 & 0 \\
C_{3} & C_{4} & C_{5} \\
C_{6} & 0 & C_{8}
\end{array}\right)\left(\begin{array}{l}
\phi_{1}(0) \\
\phi_{2}(0) \\
\phi_{3}(0)
\end{array}\right)+\left(\tau_{0}+\gamma\right)\left(\begin{array}{ccc}
0 & C_{2} & 0 \\
0 & 0 & 0 \\
0 & C_{7} & 0
\end{array}\right)\left(\begin{array}{l}
\phi_{1}(-1) \\
\phi_{2}(-1) \\
\phi_{3}(-1)
\end{array}\right),
$$

and

$$
f(\gamma, \phi)=\left(\tau_{0}+\gamma\right)\left(\begin{array}{l}
F_{1} \\
F_{2} \\
F_{3}
\end{array}\right)
$$

where

$$
\begin{aligned}
& F_{1}=-\eta \phi_{2}(-1) G^{\prime}\left(A^{*}\right) \phi_{1}(0)-\eta\left(\phi_{2}(-1)+P^{*}+k\right) \sum_{i=2}^{\infty} \frac{1}{i !} G^{i}\left(A^{*}\right) \phi_{1}(0)^{i}, \\
& F_{2}=\gamma \sum_{i=2}^{\infty} \frac{1}{i !} G^{i}\left(A^{*}\right) \phi_{1}(0)^{i}-u \phi_{2}(0) \phi_{3}(0) f^{\prime}\left(P^{*}\right)-u\left(\phi_{3}(0)+M^{*}\right) \sum_{i=2}^{\infty} \frac{1}{i !} f^{i}\left(P^{*}\right) \phi_{2}(0)^{i}, \\
& F_{3}=\alpha \sum_{i=2}^{\infty} \frac{1}{i !} f_{2}^{i}\left(P^{*}\right) \phi_{2}(-1)^{i}-\mu \phi_{3}(0) G_{1}^{\prime}\left(A^{*}\right) \phi_{1}(0)+\sum_{i=2}^{\infty} \frac{1}{i !} G_{1}^{i}\left(A^{*}\right) \phi_{1}(0)^{i}\left(\phi_{3}(0)+M^{*}\right),
\end{aligned}
$$


and $U_{t}=\phi=\left(\phi_{1}(t), \phi_{2}(t), \phi_{3}(t)\right)^{T} \in C$. Through Riesz representation theorem, there is a coefficient $3 \times 3$ matrix function $\eta(\theta, \gamma),-1 \leq \theta \leq 0$, whose elements are boundedvariation functions, and

$$
L_{\gamma}(\phi)=\int_{-1}^{0} d \eta(\theta, \gamma) \phi(\theta)
$$

We can choose

$$
\eta(\theta, \gamma)=\left(\tau_{0}+\gamma\right)\left(\begin{array}{ccc}
C_{1} & 0 & 0 \\
C_{3} & C_{4} & C_{5} \\
C_{6} & 0 & C_{8}
\end{array}\right) \delta(\theta)+\left(\tau_{0}+\gamma\right)\left(\begin{array}{ccc}
0 & C_{2} & 0 \\
0 & 0 & 0 \\
0 & C_{7} & 0
\end{array}\right) \delta(\theta+1)
$$

where $\delta(\theta)$ is the Dirac function. When $\phi \in C^{1}\left([-1,0], R^{3}\right)$, we define

$$
A(\gamma) \phi= \begin{cases}\frac{d \phi(\theta)}{d \theta}, & \theta \in[-1,0) \\ \int_{-1}^{0} d \eta(\gamma, \theta) \phi(\theta), & \theta=0\end{cases}
$$

and

$$
R(\gamma) \phi= \begin{cases}0, & \theta \in[-1,0) \\ f \eta(\gamma, \theta), & \theta=0\end{cases}
$$

Based on the previous steps, to study the Hopf bifurcation problem, we transform Eq. (11) into an operator equation of the form

$$
\dot{U}=A(\gamma)_{\gamma} U_{t}+R(\gamma)_{\gamma} U_{t}
$$

For $\phi \in C^{1}\left([0,1],\left(R^{3}\right)^{*}\right)$, define

$$
A^{*} \psi(s)= \begin{cases}-\frac{d \psi(s)}{d s}, & s \in(0,1] \\ \int_{-1}^{0} d \eta(0, s) \phi(s), & s=0\end{cases}
$$

and the bilinear inner product

$$
\langle\psi(s), \phi(\theta)\rangle=\bar{\psi}(0) \phi \cdot q(0)-\int_{-1}^{0} \int_{\xi=0}^{\theta} \bar{\psi}(\xi-\theta) d \eta(\theta) \phi(\xi) d \xi,
$$

where $\gamma=0, \eta(\theta)=\eta(\theta, 0)$, and $A(0)$ and $A^{*}(0)$ are adjoint operators. From the previous part we know that $\pm i \omega_{0} \tau_{0}$ are the eigenvalues of $A(0)$ and $A^{*}(0)$. Let $q(\theta)$ be the eigenvector of $A(0)$ corresponding to $i \omega_{0} \tau_{0}$, and let $q^{*}(s)$ be the eigenvector of $A^{*}(0)$ corresponding to $-i \omega_{0} \tau_{0}$. When $q(\theta)=\left(1, v_{1}, v_{2}\right) e^{i \omega_{0} \tau_{0} \theta}$ and $q^{*}(\theta)=\left(1, v_{1}^{*}, v_{2}^{*}\right) e^{i \omega_{0} \tau_{0} s}$, we have $A(0) q(0)=$ $i \omega_{0} \tau_{0} q(0)$ and $A^{*}(0) q^{*}(0)=-i \omega_{0} \tau_{0} q^{*}(0)$. Then we get

$$
\begin{aligned}
& q(\theta)=e^{i \omega_{0} \tau_{0} \theta}\left(1, \frac{i \omega_{0} \tau_{0}-C_{1}}{C_{2} e^{-i \omega_{0}}}, \frac{C_{2} C_{3} e^{-i \omega_{0} \tau_{0}}+\left(i \omega_{0} \tau_{0}-C_{1}\right)\left(i \omega_{0} \tau_{0}-C_{4}\right)}{C_{2} C_{5} e^{-i \omega_{0}}}\right), \\
& q^{*}(s)=G e^{-i \omega_{0} \tau_{0} s}\left(1, \frac{-i \omega_{0}-C_{1}}{C_{3}}, \frac{\left(i \omega_{0} \tau_{0}+C_{1}\right) C_{5}}{C_{3} C_{7}}-\frac{C_{8}}{C_{7}}\right)
\end{aligned}
$$


since

$$
\begin{aligned}
\left\langle q^{*}, q\right\rangle & =\bar{q}^{*}(0) \cdot q(0)-\int_{-1}^{0} \int_{\xi=0}^{\theta} \bar{q}^{*}(\xi-\theta) d \eta(\theta) q(\xi) d \xi \\
& =\bar{G}\left[\left(1+v_{1} \bar{v}_{1}^{*}+v_{2} \bar{v}_{2}^{*}\right)+\tau_{0} e^{-i \omega_{0} \tau_{0}}\left(C_{2} v_{1}+C_{6} v_{1} \bar{v}_{2}^{*}\right)\right] .
\end{aligned}
$$

To ensure $\left\langle q^{*}, q\right\rangle=1$, we get the values of $\bar{G}$ and $G$ :

$$
\begin{aligned}
& \bar{G}=\frac{1}{\left(1+v_{1} \bar{v}_{1}^{*}+v_{2} \bar{v}_{2}^{*}\right)+\tau_{0} e^{-i \omega_{0} \tau_{0}}\left(C_{2} v_{1}+C_{6} v_{1} \bar{v}_{2}^{*}\right)}, \\
& G=\frac{1}{\left(1+\bar{v}_{1} \nu_{1}^{*}+\bar{v}_{2} v_{2}^{*}\right)+\tau_{0} e^{i \omega_{0} \tau_{0}}\left(C_{2} \bar{v}_{1}+C_{6} \bar{v}_{1} v_{2}^{*}\right)} .
\end{aligned}
$$

Furthermore, the coordinates of the center manifold $C_{0}$ at $\gamma=0$ can be calculated. Let $U_{t}$ be the solution of the Eq. (11) and define

$$
z(t)=\left\langle q^{*}, x_{t}\right\rangle, \quad W(t, \theta)=U_{t}(\theta)-2 \operatorname{Re}\{z(t) q(\theta)\} .
$$

On the center manifold $C_{0}$, we have $W(t, \theta)=W(z(t), \bar{z}(t), \theta)$, where

$$
W(t, \theta)=W_{20}(\theta) \frac{z^{2}}{2}+W_{11}(\theta) z \bar{z}+W_{02}(\theta) \frac{\bar{z}^{2}}{2}+\cdots .
$$

In fact, $z(t)$ and $\bar{z}(t)$ are the local coordinates for the center manifold $C_{0}$ in the directions of $q^{*}$ and $\bar{q}^{*}$, respectively. It is worth noting that $W(t, \theta)$ is treated as a real part, and the solution of the Eq. (11) is $u_{t} \in C_{0}$. Because $\gamma=0$, by (22) we obtain

$$
\begin{aligned}
\dot{z}(t) & =i \omega_{0} \tau_{0} z(t)+\bar{q}^{*}(0) f(0, W(z, \bar{z}, \theta)+2 \operatorname{Re}\{z(t) q(\theta)\}) \\
& =i \omega_{0} \tau_{0} z(t)+\bar{q}^{*}(0) f_{0} .
\end{aligned}
$$

We rewrite it as

$$
\dot{z}(t)=i \omega_{0} \tau_{0} z(t)+g(z, \bar{z}) .
$$

According to the nature of $W$, we can define

$$
g(z, \bar{z})=g_{20} \frac{z^{2}}{2}+g_{11} z \bar{z}+g_{02} \frac{\bar{z}^{2}}{2}+g_{21} \frac{z^{2} \bar{z}}{2}+\cdots .
$$

By (22) and (23) we have

$$
\begin{aligned}
U_{t}= & W_{20}(\theta) \frac{z^{2}}{2}+W_{11}(\theta) z \bar{z}+W_{02}(\theta) \frac{\bar{z}^{2}}{2} \\
& +\left(1, v_{1}, v_{2}\right) e^{i \omega_{0} \tau_{0} \theta} z+\left(1, \bar{v}_{1}, \bar{v}_{2}\right) e^{-i \omega_{0} \tau_{0} \theta} \bar{z} .
\end{aligned}
$$

Substituting (13) and (27) into (26), we have

$$
g(z, \bar{z})=\bar{q}^{*} f_{0}(z, \bar{z})=\bar{q}^{*} f\left(0, U_{t}\right)=\bar{G} \tau_{0}\left(1, \bar{v}_{1}^{*}, \bar{v}_{2}^{*}\right)\left(\begin{array}{c}
T_{1} \\
T_{2} \\
T_{3}
\end{array}\right),
$$


where

$$
\begin{aligned}
& T_{1}=-\eta \phi_{2}(-1) G^{\prime}\left(A^{*}\right) \phi_{1}(0)-\eta\left(\phi_{2}(-1)+P^{*}+k\right) \sum_{i=2}^{\infty} \frac{1}{i !} G^{i}\left(A^{*}\right) \phi_{1}(0)^{i}, \\
& T_{2}=\gamma \sum_{i=2}^{\infty} \frac{1}{i !} G^{i}\left(A^{*}\right) \phi_{1}(0)^{i}-u \phi_{2}(0) \phi_{3}(0) f^{\prime}\left(P^{*}\right)-u\left(\phi_{3}(0)+M^{*}\right) \sum_{i=2}^{\infty} \frac{1}{i !} f^{i}\left(P^{*}\right) \phi_{2}(0)^{i}, \\
& T_{3}=\alpha \sum_{i=2}^{\infty} \frac{1}{i !} f_{2}^{i}\left(P^{*}\right) \phi_{2}(-1)^{i}-\mu \phi_{3}(0) G_{1}^{\prime}\left(A^{*}\right) \phi_{1}(0)+\sum_{i=2}^{\infty} \frac{1}{i !} G_{1}^{i}\left(A^{*}\right) \phi_{1}(0)^{i}\left(\phi_{3}(0)+M^{*}\right) .
\end{aligned}
$$

Comparing the coefficients with (26), we obtain

$$
\begin{aligned}
& g_{20}=\bar{G} \tau_{0}\left(-\bar{G} \tau_{0} \eta\left(P^{*}+k\right) G_{1}^{\prime \prime}\left(A^{*}\right)-2 \eta G_{1}^{\prime}\left(A^{*}\right) e^{-i \omega_{0} \tau_{0}} v_{1}+\left[\gamma G_{1}^{\prime \prime}\left(A^{*}\right)-2 u v_{1} v_{2} f^{\prime}\left(P^{*}\right)\right] v_{1}^{*}\right. \\
& \left.-u M^{*} f^{\prime \prime}\left(P^{*}\right) v_{1}^{*}+\left[\alpha f_{2}^{\prime \prime}\left(P^{*}\right) e^{-2 i \omega_{0} \tau_{0}} v_{1}-2 \mu v_{2}+G_{1}^{\prime \prime}\left(A^{*}\right) M^{*}\right] v_{2}^{*}\right) \text {, } \\
& g_{11}=\bar{G} \tau_{0}\left(-\bar{G} \tau_{0} \eta\left(P^{*}+k\right) G_{1}^{\prime \prime}\left(A^{*}\right)-2 \eta G_{1}^{\prime}\left(A^{*}\right)\left(v_{1}+\bar{v}_{1}\right)+\left[\gamma G_{1}^{\prime \prime}\left(A^{*}\right)-2 u\left(\bar{v}_{1} v_{2}\right) f^{\prime}\left(P^{*}\right)\right] v_{1}^{*}\right. \\
& -2 u\left(v_{1} \bar{v}_{2}\right) f^{\prime}\left(P^{*}\right) v_{1}^{*}-u M^{*} f^{\prime \prime}\left(P^{*}\right) \bar{v}_{1} v_{1}^{*}+\left[\alpha v_{1} \bar{v}_{1} f_{2}^{\prime \prime}\left(P^{*}\right)\right. \\
& \left.\left.-2 \mu\left(v_{2}+\bar{v}_{2}\right)+G_{1}^{\prime \prime}\left(A^{*}\right) M^{*}\right] v_{2}^{*}\right), \\
& g_{02}=\bar{G} \tau_{0}\left(-\bar{G} \tau_{0} \eta\left(P^{*}+k\right) G_{1}^{\prime \prime}\left(A^{*}\right)-2 \eta G_{1}^{\prime}\left(A^{*}\right) e^{i \omega_{0} \tau_{0}} \bar{\nu}_{1}+\gamma G_{1}^{\prime \prime}\left(A^{*}\right) v_{1}^{*}-2 u \bar{v}_{1} \bar{v}_{2} f^{\prime}\left(P^{*}\right) v_{1}^{*}\right. \\
& \left.-u M^{*} f^{\prime \prime}\left(P^{*}\right) v_{1}^{*}+\left[\alpha f_{2}^{\prime \prime}\left(P^{*}\right) e^{2 i \omega_{0} \tau_{0}} \bar{v}_{1}-2 \mu \bar{v}_{2}+G_{1}^{\prime \prime}\left(A^{*}\right) M^{*}\right] v_{2}^{*}\right), \\
& g_{21}=\bar{G} \tau_{0}\left(-\bar{G} \tau_{0} \eta G^{\prime}\left(A^{*}\right) \bar{\nu}_{1} e^{i \omega_{0} \tau_{0}}-\eta G^{\prime}\left(A^{*}\right) W_{20}^{(2)}(-1)-\eta W_{11}^{(1)}(0) G^{\prime \prime}\left(A^{*}\right) \bar{\nu}_{1} e^{i \omega_{0} \tau_{0}}\right. \\
& -\eta\left(P^{*}+k\right) G_{1}^{\prime \prime}\left(A^{*}\right)-\eta G^{\prime \prime}\left(A^{*}\right) \nu_{1} e^{-i \omega_{0} \tau_{0}}-\frac{2 !}{3 !} \eta\left(P^{*}+k\right) G_{1}^{\prime \prime \prime}\left(A^{*}\right) \\
& +\left[\frac{2 !}{3 !} \gamma G_{1}^{\prime \prime \prime}\left(A^{*}\right)-u f^{\prime \prime}\left(P^{*}\right) v_{1} \bar{v}_{2}-u f^{\prime \prime}\left(P^{*}\right) v_{1} \bar{v}_{1} v_{2}-\frac{2 !}{3 !} u m^{*} f^{\prime \prime \prime}\left(P^{*}\right) v_{2} \bar{v}_{2}\right. \\
& \left.-2 u M^{*} f^{\prime \prime}\left(P^{*}\right) W_{20}^{(2)}(0) \bar{v}_{1}+\gamma G_{1}^{\prime \prime}\left(A^{*}\right) W_{20}^{(1)}(0)-u W_{20}^{(2)}(0) \bar{v}_{2} f^{\prime}\left(P^{*}\right)\right] \bar{v}_{1}^{*} \\
& +\left[\frac{1}{2} \alpha f_{2}^{\prime \prime}\left(P^{*}\right) W_{20}^{(2)}(-1) v_{1} e^{-i \omega_{0} \tau_{0}}+\frac{2 !}{3 !} \alpha f_{2}^{\prime \prime \prime}\left(P^{*}\right) W_{20}^{(2)}(-1) \nu_{1} \bar{v}_{1} e^{-2 i \omega_{0} \tau_{0}}\right. \\
& -\mu\left(w_{20}^{(3)}(0)+w_{20}^{(1)}(0) \bar{v}_{2}\right)+\frac{1}{2} G_{1}^{\prime \prime}\left(A^{*}\right) M^{*} W_{20}^{(1)}(0) \\
& \left.\left.+G_{1}^{\prime \prime}\left(A^{*}\right)\left(\bar{v}_{2}+v_{2}\right)+\frac{1}{3 !} G 1^{\prime \prime \prime}\left(A^{*}\right) M^{*}\right] v_{2}^{*}\right) \text {. }
\end{aligned}
$$

Note that there are unknown quantities $W_{20}$ and $W_{11}$ in the coefficients, and we continue to calculate from the Eq. (11) and (22):

$$
\begin{aligned}
\dot{W} & =U_{t}-z \dot{q}-z \dot{\bar{q}} \\
& = \begin{cases}A(0) W-2 \operatorname{Re} q^{*}(0) f_{0} q(\theta), & \theta \in[-1,0), \\
A(0) W-2 \operatorname{Re} q^{*}(0) f_{0} q(\theta)+f_{0}, & \theta=0 .\end{cases} \\
& =A(0) W+K(z, \bar{z}, \theta),
\end{aligned}
$$


where

$$
K(z, \bar{z}, \theta)=K_{20}(\theta) \frac{z^{2}}{2}+K_{11}(\theta) z \bar{z}+K_{02}(\theta) \frac{\bar{z}^{2}}{2}+\cdots .
$$

From Eq. (23) we have

$$
\begin{aligned}
\dot{W}= & \left(W_{20}(\theta) z+W_{11}(\theta) \bar{z}+\cdots\right)\left(i \omega_{0} \tau_{0} z(t)+g(z, \bar{z})\right) \\
& +\left(W_{11}(\theta) z+W_{02}(\theta) \bar{z}+\cdots\right)\left(-i \omega_{0} \tau_{0} \bar{z}(t)+g(z, \bar{z})\right) .
\end{aligned}
$$

Through the previous calculation of (28), we get

$$
\begin{aligned}
\dot{W}= & \left(A(0) W_{20}(\theta)+K_{20}(\theta)\right) \frac{z^{2}}{2}+\left(A(0) W_{11}(\theta)\right. \\
& \left.+K_{11}(\theta)\right) z \bar{z}+\left(A(0) W_{02}(\theta)+K_{02}(\theta)\right)+\cdots .
\end{aligned}
$$

Comparing the coefficients of (30) and (31), we obtain

$$
\left(A(0)-2 i \omega_{0} \tau_{0}\right) W_{20}(\theta)=-K_{20}(\theta), \quad A(0) W_{11}(\theta)=-K_{11}(\theta) .
$$

Based on (28), when $\theta \in[-1,0)$, we get

$$
\begin{aligned}
K(z, \bar{z}, \theta)= & -\left(g_{20} \frac{z^{2}}{2}+g_{11} z \bar{z}+g_{02} \frac{\bar{z}^{2}}{2}+g_{21} \frac{z^{2} \bar{z}}{2}+\cdots\right) q(\theta) \\
& -\left(\bar{g}_{20} \frac{\bar{z}^{2}}{2}+\bar{g}_{11} z \bar{z}+\bar{g}_{02} \frac{z^{2}}{2}+\bar{g}_{21} \frac{\bar{z}^{2} z}{2}+\cdots\right) \bar{q}(\theta) .
\end{aligned}
$$

The coefficients at $z^{2}$ and $z \bar{z}$ are

$$
K_{20}(\theta)=-g_{20} q(\theta)-\bar{g}_{02} \bar{q}(\theta)
$$

and

$$
K_{11}(\theta)=-g_{11} q(\theta)-\bar{g}_{11} \bar{q}(\theta) .
$$

From (32) and (34) we get

$$
A(0) W_{20}(\theta)=2 i \omega_{0} \tau_{0} W_{20}(\theta)-H_{20}(\theta) \text {. }
$$

By the definition of $A(0)$ we have

$$
\dot{W}_{20}(\theta)=2 i \omega_{0} \tau_{0} W_{20}(\theta)+g_{2} 0 q(\theta)+\bar{g}_{0} 2 \bar{q}(\theta) .
$$

Note that $q(\theta)=q(0) e^{i \omega_{0} \tau_{0} \theta}$, and therefore

$$
W_{20}(\theta)=\frac{i g_{20}}{\omega_{0} \tau_{0}} q(0) e^{i \omega_{0} \tau_{0} \theta}+\frac{i \bar{g}_{02}}{\omega_{0} \tau_{0}} \bar{q}(0) e^{-i \omega_{0} \tau_{0} \theta}+D_{1} e^{2 i \omega_{0} \tau_{0} \theta},
$$


where $D_{1}=\left(D_{1}^{(1)}, D_{1}^{(2)}, D_{1}^{(3)}\right)^{T}$ is a constant factor, and from (32) and (35) we obtain

$$
\dot{W}_{11}(\theta)=g_{11} q(\theta)+\bar{g}_{11} \bar{q}(\theta)
$$

and

$$
W_{11}(\theta)=-\frac{i g_{11}}{\omega_{0} \tau_{0}} q(0) e^{i \omega_{0} \tau_{0} \theta}+\frac{i \bar{g}_{11}}{\omega_{0} \tau_{0}} \bar{q}(0) e^{-i \omega_{0} \tau_{0} \theta}+D_{2},
$$

where $D_{2}=\left(D_{2}^{(1)}, D_{2}^{(2)}, D_{2}^{(3)}\right)^{T}$ is a constant factor. We can find approximate values of $D_{1}$ and $D_{2}$ in (36) and (37), respectively. It follows from the form defined by $A$ and (32) that

$$
\int_{-1}^{0} d \eta(\theta) W_{20}(\theta)=2 i \omega_{0} W_{20}(\theta)-K_{20}(\theta)
$$

and

$$
\int_{-1}^{0} d \eta(\theta) W_{11}(\theta)=-K_{11}(\theta)
$$

where $\eta(\theta)=\eta(0, \theta)$. From (28) we have

$$
\begin{aligned}
& K_{20}(0)=-g_{20} q(\theta)-\bar{g}_{02} \bar{q}(\theta)+\tau_{0}\left(\begin{array}{l}
R_{11} \\
R_{12} \\
R_{13}
\end{array}\right), \\
& R_{11}=-\bar{G} \tau_{0} \eta\left(P^{*}+k\right) G_{1}^{\prime \prime}\left(A^{*}\right)-2 \eta G_{1}^{\prime}\left(A^{*}\right) e^{-i \omega_{0} \tau_{0}} v_{1}, \\
& R_{12}=\gamma G_{1}^{\prime \prime}\left(A^{*}\right)-2 u v_{1} v_{2} f^{\prime}\left(P^{*}\right)-u M^{*} f^{\prime \prime}\left(P^{*}\right), \\
& R_{13}=\alpha f_{2}^{\prime \prime}\left(P^{*}\right) e^{-2 i \omega_{0} \tau_{0}} v_{1}-2 \mu v_{2}+G_{1}^{\prime \prime}\left(A^{*}\right) M^{*},
\end{aligned}
$$

and

$$
K_{11}(0)=-g_{11} q(\theta)-\bar{g}_{11} \bar{q}(\theta)+2 \tau_{0}\left(\begin{array}{l}
R_{21} \\
R_{22} \\
R_{23}
\end{array}\right) \text {, }
$$

where

$$
\begin{aligned}
& R_{21}=-\bar{G} \tau_{0} \eta\left(P^{*}+k\right) G_{1}^{\prime \prime}\left(A^{*}\right)-2 \eta G_{1}^{\prime}\left(A^{*}\right) v_{1} \bar{v}_{1}, \\
& R_{22}=\left[\gamma G_{1}^{\prime \prime}\left(A^{*}\right)-2 u\left(\bar{v}_{1} v_{2}+v_{1} \bar{v}-2\right) f^{\prime}\left(P^{*}\right)-u M^{*} f^{\prime \prime}\left(P^{*}\right) \bar{v}_{1}\right] v_{1}^{*}, \\
& R_{23}=\left[\alpha v_{1} \bar{v}_{1} f_{2}^{\prime \prime}\left(P^{*}\right)-2 \mu\left(v_{2}+\bar{v}_{2}\right)+G_{1}^{\prime \prime}\left(A^{*}\right) M^{*}\right] v_{2}^{*} .
\end{aligned}
$$

Because $i \omega_{0} \tau_{0}$ is an eigenvalue of $A(0)$ and $q(0)$, we have

$$
\left(i \omega_{0} \tau_{0} I-\int_{-1}^{0} e^{i \omega_{0} \tau_{0} \theta} d \eta(\theta)\right) q(0)=0
$$


and

$$
\left(-i \omega_{0} \tau_{0} I-\int_{-1}^{0} e^{-i \omega_{0} \tau_{0} \theta} d \eta(\theta)\right) \bar{q}(0)=0 .
$$

Therefore we obtain

$$
\left(\begin{array}{ccc}
2 i \omega_{0}-C_{1} & -C_{2} & 0 \\
-C_{3} & 2 i \omega-C_{4} & -C_{5} \\
-C_{6} & -C_{7} & 2 i \omega-C_{8}
\end{array}\right) \times D_{1}=\left(\begin{array}{l}
R_{11} \\
R_{12} \\
R_{13}
\end{array}\right)
$$

and

$$
\left(\begin{array}{ccc}
-C_{1} & -C_{2} & 0 \\
-C_{3} & -C_{4} & -C_{5} \\
-C_{6} & -C_{7} & -C_{8}
\end{array}\right) \times D_{2}=\left(\begin{array}{l}
R_{21} \\
R_{22} \\
R_{23}
\end{array}\right)
$$

From (36), (37), (44), and (45), we can express $g_{21}$. Furthermore, we can calculate the following values:

$$
\begin{aligned}
& C_{1}(0)=\frac{i}{2 \omega_{0} \tau_{0}}\left(g_{11} g_{20}-2\left|g_{11}\right|^{2}-\frac{\left|g_{02}\right|^{2}}{3}\right)+\frac{g_{21}}{2}, \\
& \mu_{2}=\frac{\operatorname{Re}\left(C_{1}(0)\right)}{\operatorname{Re}\left(\lambda^{\prime}\left(\tau_{0}\right)\right)} \\
& T_{2}=-\frac{\operatorname{Im} C_{1}(0)+\mu_{2} \operatorname{Im} \lambda^{\prime}\left(\tau_{0}\right)}{\omega_{0} \tau_{0}}, \\
& \beta_{2}=2 \operatorname{Re} C_{1}(0) .
\end{aligned}
$$

According to the Hassard theory, we can obtain the following theorem.

Theorem 4.1 The bifurcating periodic solution of (11) from the trivial equilibrium has the following features:

(1) $\mu_{2}$ determines the direction of the Hopf bifurcation: if $\mu_{2}>0\left(\mu_{2}<0\right)$, then the Hopf bifurcation is supercritical (subcritical), and the bifurcating periodic solutions exist for $\tau>\tau_{0}\left(\tau<\tau_{0}\right)$;

(2) $\beta_{2}$ determines the stability of the bifurcating periodic solutions: the bifurcating periodic solutions are stable (unstable) if $\beta_{2}<0\left(\beta_{2}>0\right)$;

(3) $T_{2}$ determines the period of the bifurcating periodic solutions: the period increases (decreases) if $T_{2}>0\left(T_{2}<0\right)$.

\section{Numerical simulation}

The numerical calculation is carried out by using the software Matlab. We choose the same proportion set of parameters as those in [9]: $\beta=0.006, \eta=0.04, u=0.14, \mu=0.01$, $\alpha=0.0048, \gamma=0.004, k_{1}=1, k_{2}=1, k_{3}=0.1, k_{4}=1, k=0.1$. We can obtain that the steadystate solution of the equation is $\hat{E}(0.352,0.476,0.090)$. By using the derivation in Sect. 3 we conclude that the critical value of Hopf bifurcation $\tau_{0}$ is equal to 23.237. From the conclusion of Sect. 4 we find that the obtained Hopf bifurcation point is supercritical, and 


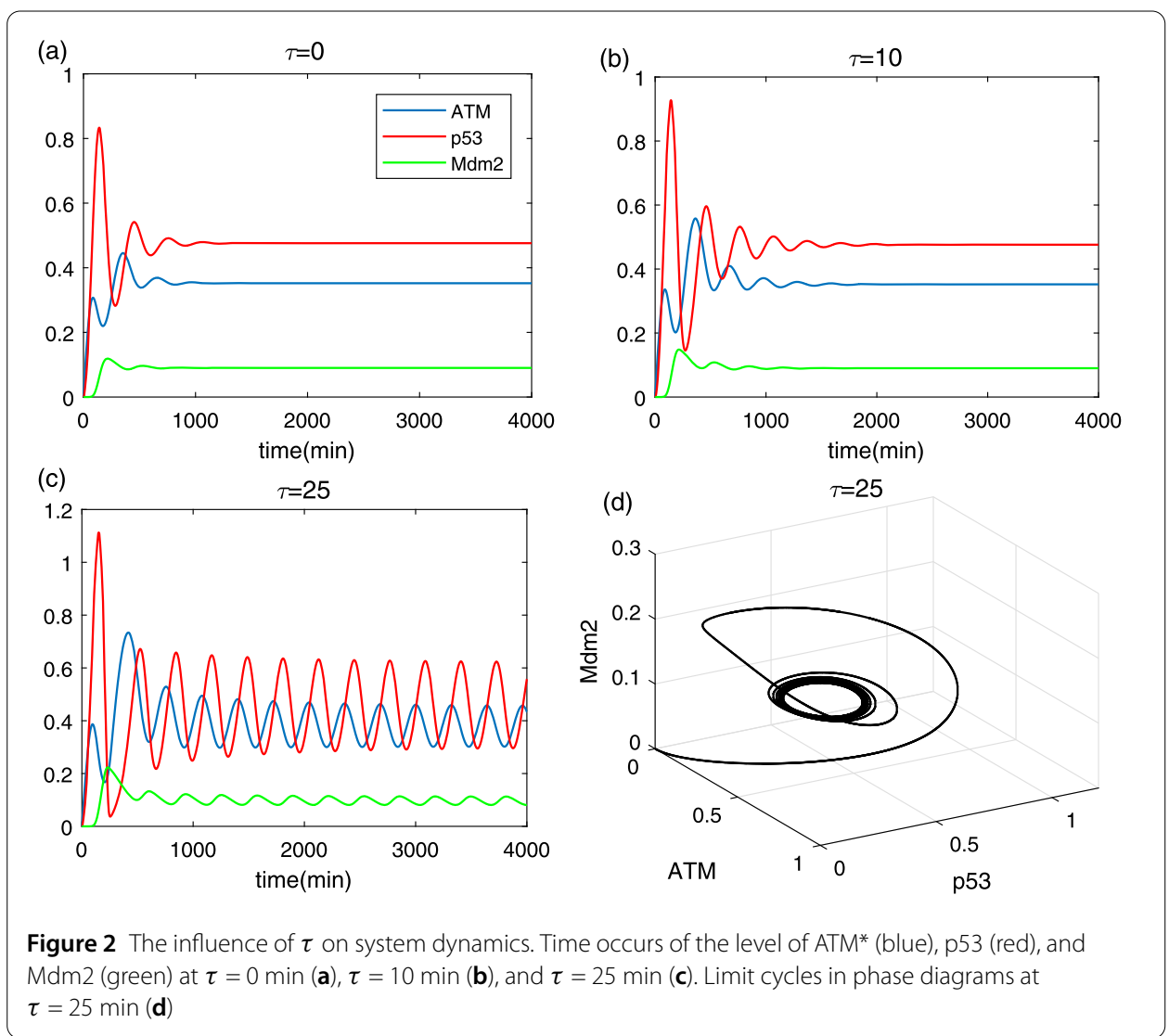

the period of oscillation increases with the increase of $\tau$. Next, we use a time evolution diagram and bifurcation diagram to illustrate our theoretical results.

The units of time is minute, and the units of other parameters ensure that the concentrations of proteins are dimensionless. The initial values of the system are $A(0)=P(0)=$ $M(0)=0$. The numerical simulation results of time occurs are shown in Fig. 2. When $\tau$ is equal to 0 or $10\left(<\tau_{0}\right)$ in Fig. 2(a) or (b), the system has damping oscillations and finally tends to steady state. When the value of $\tau$ is $25\left(>\tau_{0}\right)$ in Fig. 2(c), the system has stable oscillations with a period of 4-7 hours [30]. Therefore the time delay is a necessary condition for p53 to oscillate. The phase diagram in Fig. 2(d) shows that the system finally converges to a stable limit cycle.

We further draw a bifurcation diagram to study the effect of $\tau$ on the p53 pulse. The functional relationship between p53 level and time delay $\tau$ is displayed in bifurcation graph Fig. 3(a). The coordinates of the Hopf bifurcation point are $(23.237,0.476)$. With the increase of $\tau$, the steady state loses its stability. After the supercritical Hopf bifurcation point, system enters the oscillation state. With the increase of $\tau$, the amplitude first sharply increases and then slowly increases. In Fig. 3(b), the relationship between the period of the oscillation and the time delay $\tau$ is almost linear after the Hopf bifurcation point. The results of numerical simulation are consistent with those of our theoretical analysis.

In addition, we theoretically analyze the network dynamics in the deterministic case. Noise always exists in biological systems [20,21], and it is essential to explore the effect of noise on system dynamics. We use the multiplier exponential Gaussian white noise, that is, $\zeta=\exp (\epsilon)$, where $\epsilon$ obeys the Gaussian distribution with mean 0 and standard deviation $D$, 

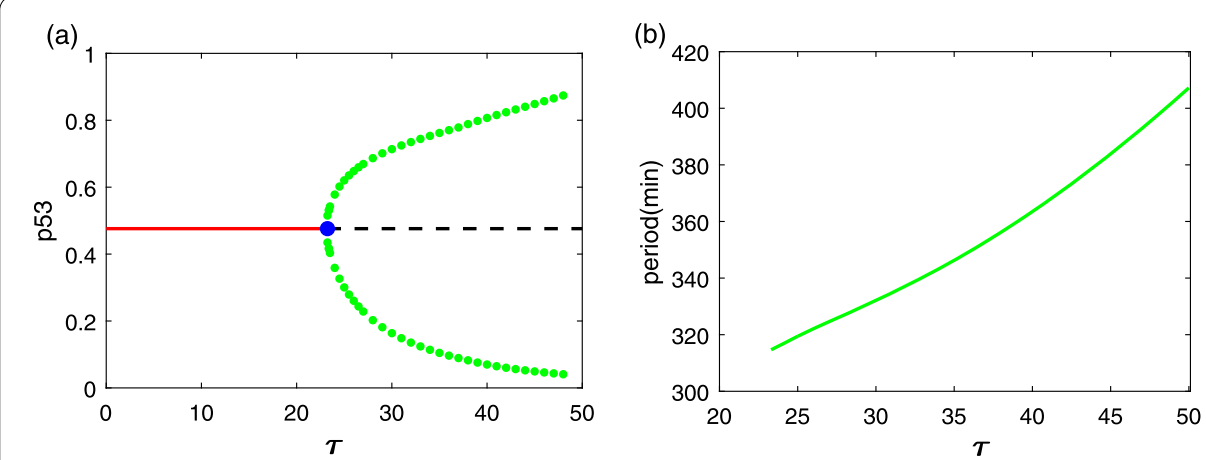

Figure $3 \tau$ regulates p53 pulse. (a) Bifurcation diagram of p53 level vs. $\tau$. The red solid line represents stable steady state. The black dashed line represents unstable steady state. The green point represents the peak or trough of p53 oscillation, and the blue point represents the supercritical Hopf bifurcation point. (b) The period of p53 oscillation as a function of $\tau$
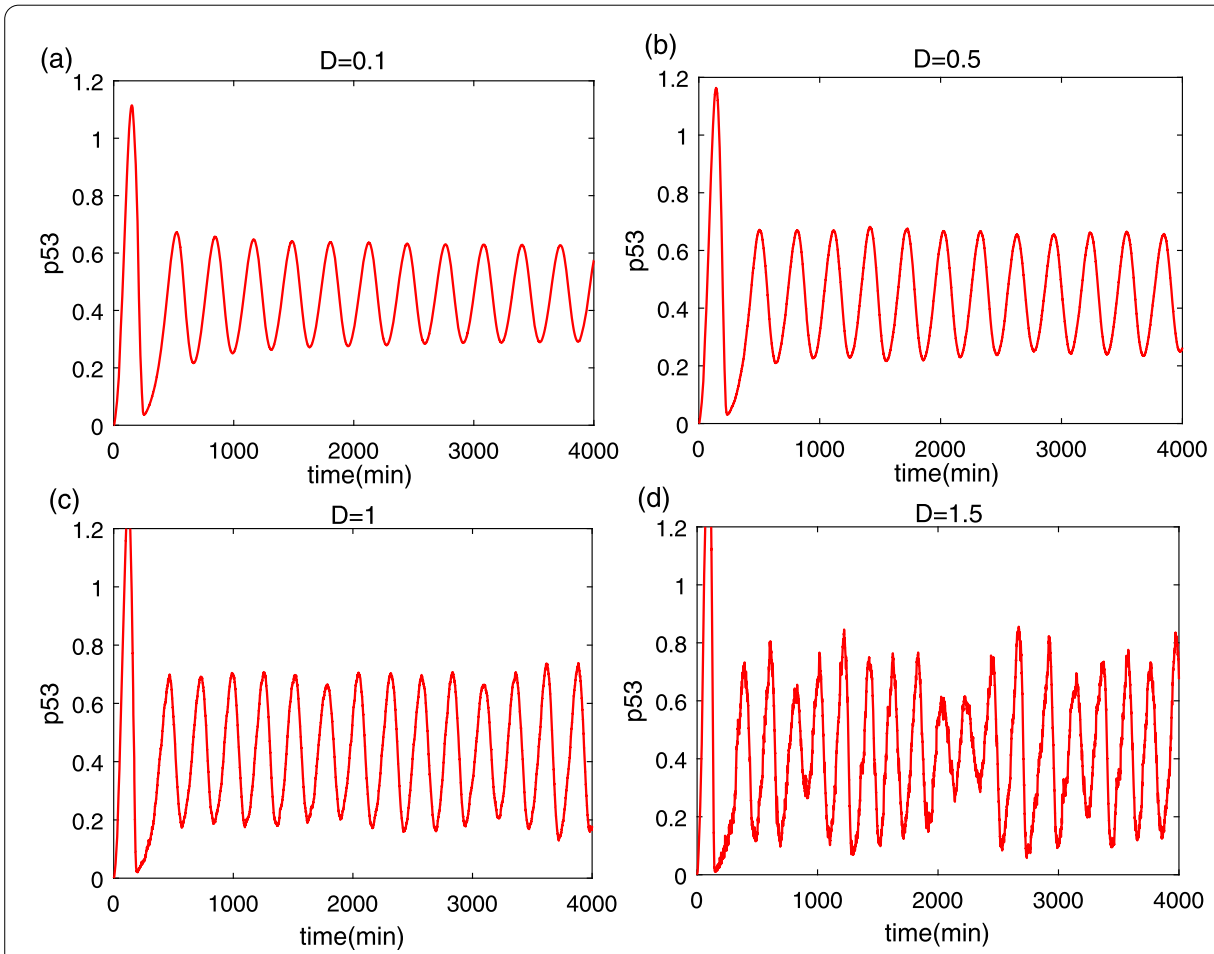

Figure 4 The period of p53 pulse is more stable than the amplitude in noise environment. Time occurs of the level of p53 at $\tau=25$ and noise intensity $D=0.1(\mathbf{a}), D=0.5(\mathbf{b}), D=1(\mathbf{c})$, and $D=1.5$ (d)

which characterize the noise intensity; we write this as $\epsilon \sim N(0, D)$. The system oscillation is stable under weak noise in Figs. 4(a), 4(b), and 4(c), which indicate that at moderate noise intensity, the period of oscillation is stable. Figure 4(d) implies that at high noise intensity, the system amplitude is unstable. Our numerical simulation is consistent with experimental results, that is, the period of the p53 pulse is more robust than the amplitude, and not all cells have stable pulses [30]. 


\section{Discussion}

In this paper, we constructed a minimodel to explore how time delay affected the oscillation behavior of $\mathrm{p} 53$ in response to DNA damage. We find that if time delay $\tau$ is greater than $\tau_{0}$, then the system is oscillatory; otherwise, it is asymptotically stable. We also use the normal form method and the central manifold theorem to further analyze the effect of parameter $\tau$ on the kinetics of p53 and predict the direction and stability of the bifurcation diagram.

The correctness of the theory is verified by numerical simulation. The bifurcation diagram of p53 level versus $\tau$ vividly shows that the oscillation of p53 can be regulated by changing the parameter $\tau$. Our model indicates that the effect of noise on the amplitude is more severe than the period. Because the p53 pulse is closely related to cell fate decision, it may be a good idea to consider the time delay in the treatment of cancer.

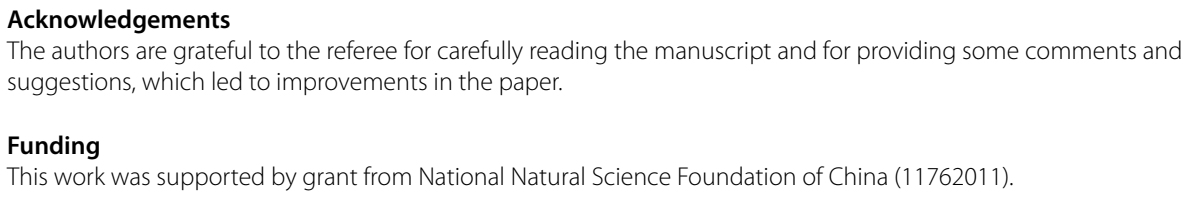

\section{Publisher's Note}

Springer Nature remains neutral with regard to jurisdictional claims in published maps and institutional affiliations.

Received: 3 February 2020 Accepted: 24 June 2020 Published online: 08 July 2020

References

1. Murray, F.Z., Slee, E.A., Lu, X.: A complex barcode underlies the heterogeneous response of p53 to stress. Nat. Rev. Mol. Cell Biol. 9, 702-712 (2008)

2. Wu, X., Bayle, J.H., Olson, D., Levine, A.J.: The p53-mdm2 autoregulatory feedback loop. Genes Dev. 7, 1126-1132 (1993)

3. Vousden, K.H., Prives, C.: Blinded by the light: the growing complexity of p53. Cell 137, 137413-137431 (2009)

4. Siliciano, J.D., Canman, C.E., Taya, Y., Sakaguchi, K., Appella, E., Kastan, M.B.: DNA damage induces phosphorylation of the amino terminus of p53. Genes Dev. 11, 3471-3481 (1997)

5. Sun, T.Z., Yang, W., Liu, J., Shen, P.: Modeling the basal dynamics of P53 system. PLoS ONE 6, e27882 (2011)

6. Ma, L., Wagner, J., Jeremy, J., Hu, R.W., Levine, A.J., Stolovitzky, G.A.: A plausible model for the digital response of p53 to DNA damage. Proc. Natl. Acad. Sci. USA 102, 14266-14271 (2005)

7. Zhang, T.L., Brazhnik, P., Tyson, J.J.: Exploring mechanisms of the DNA-damage response p53 pulses and their possible relevance to apoptosis. Cell Cycle 6, 85-94 (2007)

8. Zhang, X.P., Liu, F., Cheng, Z., Wang, W.: Cell fate decision mediated by p53 pulses. Proc. Natl. Acad. Sci. USA 106 $12245-12250$ (2009)

9. Zhang, X.P., Liu, F.: Two-phase dynamics of p53 in the DNA damage response. Proc. Natl. Acad. Sci. USA 22, 8990-8995 (2011)

10. Sun, T.Z., Chen, C., Wu, Y.Y., Zhang, S.: Modeling the role of p53 pulses in DNA damage-induced cell death decision. BMC Bioinform. 10, 190 (2009)

11. Hat, B., Marek, K., Marta, B.N., Lipniacki, T.: Feedbacks, bifurcations and cell fate decision-making in the p53 system. PLoS Comput. Biol. 12, e1004787 (2016)

12. Aylon, Y., Oren, M.: Living with p53, dying of p53. Cell 130, 597 (2007)

13. Purvis, J.E., Karhohs, K.W., Mock, C., Batchelor, E., Loewer, A., Lahav, G.: p53 dynamics control cell fate. Science 366, 1440-1444 (2012)

14. Lahav, G., Rosenfeld, N., Sigal, A., Zatorsky, N.G., Levine, A.J., Elowitz, M.B.: Dynamics of the p53-Mdm2 feedback loop in individual cells. Nat. Genet. 36, 147-150 (2004)

15. Puszynski, K., Hat, B., Lipniacki, T.: Oscillations and bistability in the stochastic model of p53 regulation. Theor. Biol. $254,452-465(2008)$ 
16. Batchelor, E., Mock, C.S., Bhan, I., Loewer, A., Lahav, G.: Recurrent initiation: a mechanism for triggering p53 pulses in response to DNA damage. Mol. Cell 30, 277-289 (2008)

17. Lahav, G.: The strength of indecisiveness: oscillatory behavior for better cell fate determination. Sci. STKE 264,55 (2004)

18. Ciliberto, A., Novak, B., Tyson, J.J.: Steady states and oscillations in the p53/Mdm2 network. Cell Cycle 4, 488-493 (2005)

19. Fang, H.P., Hu, L., Wu, Y.F.: Delay-induced Hopf bifurcation in a diffusive Holling-Tanner predator-prey model with ratio-dependent response and Smith growth. Adv. Differ. Equ. 2018, 285 (2018)

20. Lewism, J.: From signals to patterns: space, time, and mathematics in developmental biology. Science 322, 399-403 (2008)

21. Yalamanchili, H.K., Yan, B., Li, M.J., Qin, J., Zhao, Z.Y., Chin, F.Y.L., Wang, J.W.: Dynamic delay gene-network inference from high-temporal data using gapped local alignment. Bioinformatics 30, 377-383 (2014)

22. Prindle, A., Selimkhanov, J., Li, H., Razinkov, I., Tsimring, L.S., Hasty, J.: Rapid and tunable post-translational coupling of genetic circuits. Nature 508, 387-391 (2014)

23. Antti, H., Jaakko, P., Hande, T., Iryna, C., Filomena, M., Korbinian, G., Stunnenberg, H.G., George, R., Lawrence, N.D., Magnus, R.: Genome-wide modeling of transcription kinetics reveals patterns of RNA production delays. Proc. Natl. Acad. Sci. USA 112, 13115-13120 (2015)

24. Zhang, Z.Z., Wang, Y.G.: Hopf bifurcation of a heroin model with time delay and saturated treatment function. Adv. Differ. Equ. 2019, 64 (2019)

25. Zhang, Y., Wang, C.H., Yan, F., Liu, H.H.: Theoretical study on the oscillation mechanism of p53-Mdm2 network. Int. J. Biomath. 11, 22 (2018)

26. Haupt, Y., Maya, R., Kazaz, A., Oren, M.: Mdm2 promotes the rapid degradation of p53. Nature 387, 296 (1997)

27. Prives, C.: Signaling to p53: breaking the MDM2-p53 circuit. Cell 95, 5-8 (1998)

28. Shreeram, S., Demidov, O.N., Hee, W.K., Yamaguchi, H., Bulavin, D.V.: Wip1 phosphatase modulates ATM-dependent signaling pathways. Mol. Cell 23, 757-764 (2006)

29. Mayo, L.D., Donner, D.B.: A phosphatidylinositol 3-kinase/Akt pathway promotes translocation of Mdm2 from the cytoplasm to the nucleus. Proc. Natl. Acad. Sci. USA 98, 11598-11603 (2001)

30. Zatorsky, N.G., Rosenfeld, N., Itzkovitz, S., Milo, R., Sigal, A.: Oscillations and variability in the p53 system. Mol. Syst. Biol. $2,0033(2006)$

\section{Submit your manuscript to a SpringerOpen ${ }^{\circ}$ journal and benefit from:}

- Convenient online submission

- Rigorous peer review

- Open access: articles freely available online

- High visibility within the field

- Retaining the copyright to your article

Submit your next manuscript at $\boldsymbol{~ s p r i n g e r o p e n . c o m ~}$ 